\title{
Variabilidade da composição nutricional de coprodutos de etanol de milho do Brasil para suínos
}

\author{
Variability of the nutritional composition of Brazilian corn ethanol coproducts for pigs \\ Variabilidad de la composición nutricional de los coproductos de etanol de maíz de Brasil para \\ cerdos
}

\section{Resumo}

A indústria de etanol a partir de grãos vem crescendo no Brasil devido a disponibilidade de milho gerando grande diversidade e quantidade de coprodutos para alimentação animal. Esses coprodutos podem ser ingredientes para a indústria suinícola desde que se conheça sua composição. Objetivou-se referenciar a composição química e nutricional para suínos de coprodutos brasileiros. Foram realizadas determinações de matéria seca (MS), matéria mineral (MM), proteína bruta (PB), extrato etéreo (EE), fibra em detergente neutro (FDN), tamanho de partículas (TP), e energia bruta (EB), digestível (ED), metabolizável (EM), líquida (EL) além de lisina total (LT) e digestível (LD) via equações de predição de nove coprodutos de etanol de milho. Houve expressiva variação na composição química e física dos coprodutos brasileiros analisados o que influenciou em variabilidade nos valores de energia e lisina. As maiores amplitudes de valores foram registrados para MS, MM, EB, LT e LD. Os maiores valores para PB foram registrados no HP-DDGS seguido pelos DDG's da U2 e da U4, enquanto os menores valores de MS e FDN foram encontrados nos solúveis. Os maiores valores de ED, EM e EL foram registradas no HP-DDGS, WDG e DDGS da usina U1. O HP-DDGS apresentou a melhor composição química, física e nutricional sugerindo maior aptidão para uso em dietas para suínos.

Palavras-chave: DDGS; Energia líquida; Energia metabolizável; HP-DDGS; Lisina digestível. 


\begin{abstract}
The grain ethanol industry has been growing in Brazil due to the availability of corn, generating great diversity and quantity of by-products for animal feed. These co-products can be ingredients for the pig industry as long as their composition is known. The objective was to reference the chemical and nutritional composition for pigs from Brazilian by-products. Determinations of dry matter (DM), mineral matter (MM), crude protein (PB), ether extract (EE), neutral detergent fiber (NDF), particle size (TP), and gross energy (EB) were carried out, digestible (ED), metabolizable (EM), liquid (EL) in addition to total lysine (LT) and digestible (LD) via prediction equations for nine corn ethanol by-products. There was a significant variation in the chemical and physical composition of the Brazilian co-products analyzed, which influenced the variability in the values of energy and lysine. The highest amplitudes of values were recorded for MS, MM, EB, LT and LD. The highest values for PB were registered in the HP-DDGS followed by the DDGs of U2 and U4, while the lowest values of MS and NDF were found in the soluble ones. The highest values of ED, EM and EL were registered in the HP-DDGS, WDG and DDGS of the U1 plant. The HP-DDGS showed the best chemical, physical and nutritional composition, suggesting greater suitability for use in pig diets.
\end{abstract}

Keywords: DDGS; Digestible lysine; HP-DDGS; Metabolizable energy; Net energy.

\title{
Resumen
}

La industria del etanol en grano ha venido creciendo en Brasil debido a la disponibilidad de maíz, generando gran diversidad y cantidad de subproductos para la alimentación animal. Estos coproductos pueden ser ingredientes para la industria porcina siempre que se conozca su composición. El objetivo fue referenciar la composición química y nutricional de los cerdos a partir de subproductos brasileños. Se realizaron determinaciones de materia seca (MS), materia mineral $(\mathrm{MM})$, proteína bruta $(\mathrm{PB})$, extracto etéreo (EE), fibra detergente neutra (FDN), tamaño de partícula (TP) y energía bruta (EB) digestible (ED), metabolizable (EM), líquido (EL) además de lisina total (LT) y digestible (LD) mediante ecuaciones de predicción para nueve subproductos de etanol de maíz. Hubo una variación significativa en la composición química y física de los coproductos brasileños analizados, lo que influyó en la variabilidad en los valores de energía y lisina. Las mayores amplitudes de valores se registraron para MS, MM, EB, LT y LD. Los valores más altos de PB se registraron en los HP-DDGS seguidos de los DDG de U2 y U4, mientras que los valores más bajos de MS y NDF se encontraron en los solubles. Los valores más altos de ED, EM y EL se registraron en los HP-DDGS, WDG y DDGS de la planta U1. El HP-DDGS mostró la mejor composición química, física y nutricional, sugiriendo una mayor idoneidad para su uso en dietas porcinas.

Palabras clave: DDGS; Energía metabolizable; Energía neta; HP-DDGS; Lisina digestible.

\section{Introdução}

Os biocombustíveis são alternativas ao petróleo desde 1970 devido à preocupação com a poluição ambiental e a emissão de gases de efeito estufa que são gerados pelo combustível fóssil (Leite \& Leal, 2007). Os Estados Unidos e o Brasil respondem pelos maiores volumes de etanol no mundo (RFA, 2020), mas usando matérias primas distintas. Uma expressiva mudança vem ocorrendo em locais como no estado de Mato Grosso cuja disponibilidade de grãos é abundante e a instalação de usinas de etanol de milho gera quantitativos próximos daquelas a base de cana-de-açúcar (CONAB, 2020), culminando em oportunidade econômica para o país (Silva \& Castañeda-Ayarza, 2021).

A produção de etanol a partir de grãos gera diversos coprodutos entre esses, os grãos secos de destilaria com solúveis (DDGS), cujo rendimento é de $32,3 \mathrm{~kg}$ a cada $100 \mathrm{~kg}$ de milho além de 40,2 L de etanol e $32,3 \mathrm{~kg}$ de $\mathrm{CO}_{2}(\mathrm{Chatzifragkou}$ et al., 2015), gerando um volume expressivo de coprodutos. Contudo, ampla diversidade de coprodutos pode ser produzida com características químicas, físicas e nutricionais que repercutem em seu uso enquanto ingredientes para alimentação animal. Como $60 \%$ a $70 \%$ do grão de milho é amido, quando este é convertido em etanol durante a fermentação, as concentrações de proteínas, lipídios e fibras são aumentadas (Stein, 2008). Tipicamente o DDGS apresenta cerca de 30\% de proteína, 10\% de gordura e $40 \%$ de fibra em detergente neutro (Rosentrater et al., 2019).

Com enorme potencial de substituição aos ingredientes tradicionais, os coprodutos de etanol de milho podem reduzir custos e otimizar formulações de rações para cadeias produtivas como a suinocultura brasileira, a quinta maior do mundo (ABPA, 2019) entretanto, pouquíssimos estudos com coprodutos do Brasil foram realizados (Corassa et al., 2017, 2019, 2021). A inclusão de DDGS na dieta pode proporcionar redução nos custos nutricionais e melhor a rentabilidade da produção dos suínos (Fantini et al., 2021) contudo, diferenças nas matérias primas (Anderson et al., 2012, Wu et al., 2016a) e processos de fabricação (Böttger \& Südekum, 2018) constroem a hipótese que os coprodutos locais distinguem-se entre si e com os de outros 
países. Logo, a caracterização das propriedades dos coprodutos brasileiros se faz necessária para o adequado uso destes em dietas para suínos.

Assim, objetivou-se com este estudo referenciar a composição química, física e nutricional de coprodutos da produção de etanol de usinas brasileiras destinados à alimentação de suínos.

\section{Metodologia}

Foram coletadas amostras de grãos úmidos de destilaria (wet distillers grains, WDG), grãos secos de destilaria (distillers dried grains, DDG), solúveis (solubles), grãos secos de destilaria com solúveis (distillers dried grains with solubles, DDGS) e grãos secos de destilaria com solúveis de alta proteína (high protein distillers dried grains with solubles, HP-DDGS) oriundos de usinas produtoras de etanol de milho localizadas no estado de Mato Grosso (Tabela 1).

Tabela 1 - Descrição do produto com sua respectiva origem.

\begin{tabular}{lll}
\hline Usina & Produto & Município \\
\hline Usina 1 (U1) & HP-DDGS, DDGS, WDG & Lucas do Rio Verde - MT \\
Usina 2 (U2) & WDG, DDG, solúveis & Sorriso - MT \\
Usina 3 (U3) & DDG, WDG & São José do Rio Claro - MT \\
Usina 4 (U4) & DDG & Campos de Júlio - MT \\
\hline
\end{tabular}

Fonte: Autores (2021).

Os coprodutos foram encaminhados ao Laboratório de Nutrição de Monogástricos do Campus Universitário de Sinop da Universidade Federal de Mato Grosso para sub-amostragem e realização das análises químicas, físicas e nutricionais. Determinou-se o tamanho de partícula (TP) dos coprodutos através de conjunto de peneiras (Zanotto \& Bellaver, 1996). Amostras de cada coproduto foram moídas com peneira de $1.0 \mathrm{~mm}$ para determinação dos teores de matéria seca (MS - método 930.15), matéria mineral (MM - método 923.03), proteína bruta (PB- método 954.01), extrato etéreo (EE- método 991.36), fibra insolúvel em detergente neutro (FDN- método 2002.04) e fósforo inorgânico total (P- método 945.38) segundo AOAC (2005) enquanto a energia bruta (EB) foi determinada por calorimetria (Parr 6400).

Os valores de energia bruta (EB), digestível (ED), metabolizável (EM) e líquida (EL) foram estimadas segundo as equações: $\mathrm{EB}=(4.583+(50,6 *$ extrato etéreo $)-(0.1 *$ tamanho de partícula $))($ Kerr et al., 2013$) ; \mathrm{ED}=(-2.161+(1,39 *$ energia bruta) (20,7*fibra em detergente neutro)-(49,3*extrato etéreo)) (Anderson et al., 2012); EM=(-261+(1,05*energia digestível)$(7,89 *$ proteína bruta $)+(2,47 *$ fibra em detergente neutro)-(4,99*extrato etéreo)) (Anderson et al., 2012$)$; e EL = -1130.5 + $(0.727 \times \mathrm{EB})+(23.86 \times \mathrm{EE})-(10.83 \times \mathrm{FDN})(\mathrm{Wu}$ et al., 2016b). As equações de EB, ED e EM foram avaliadas e validadas por Urriola et al. (2014).

Os teores de lisina total dos coprodutos foram calculados utilizando os valores de PB determinados em laboratório e a relação de lisina:proteína (2,97), enquanto os teores de lisina digestível foram calculados utilizado o coeficiente de digestibilidade (0,617), ambas informações obtidas da meta-análise de Zeng et al. (2017).

Os dados foram submetidos à comparação estatística seguindo o delineamento inteiramente casualizado (DIC) com cada coproduto caracterizando um tratamento e três repetições por coproduto utilizando procedimento GLM do programa SAS (SAS Institute, Inc, Cary, NC, USA) para análises de variância e comparação de médias pelo Teste de Tukey à 0,01. 


\section{Resultados e Discussão}

Houve expressiva variação na composição química e física dos coprodutos brasileiros analisados (Tabela 2), o que influenciou em variabilidade nos valores de energia (Tabela 3) e lisina (Figura 1) destes ingredientes.

Tabela 2 - Matéria seca (MS), matéria mineral (MM), proteína bruta (PB), extrato etéreo (EE), fibra em detergente neutro (FDN) e tamanho de partícula (TP) de diferentes fontes de coprodutos.

\begin{tabular}{cccccccc}
\hline Produto & Origem & MS $(\%)$ & MM $(\%)$ & PB $(\%)$ & EE $(\%)$ & FDN $(\%)$ & TP $(\mu \mathrm{m})$ \\
\hline WDG & U1 & $40,90^{\mathrm{d}}$ & $7,72^{\mathrm{a}}$ & $23,30^{\mathrm{d}}$ & $6,56^{\mathrm{b}}$ & $26,54^{\mathrm{c}}$ & $1285^{\mathrm{a}}$ \\
WDG & $\mathrm{U} 2$ & $30,68^{\mathrm{e}}$ & $1,09^{\mathrm{g}}$ & $26,80^{\mathrm{d}}$ & $3,44^{\mathrm{c}}$ & $54,36^{\mathrm{ab}}$ & $87^{\mathrm{d}}$ \\
WDG & $\mathrm{U} 3$ & $28,13^{\mathrm{f}}$ & $1,52^{\text {ef }}$ & $39,76^{\mathrm{bc}}$ & $6,12^{\mathrm{bc}}$ & $50,16^{\mathrm{ab}}$ & $966^{\mathrm{c}}$ \\
DDG & $\mathrm{U} 3$ & $93,82^{\mathrm{a}}$ & $1,62^{\mathrm{e}}$ & $35,72^{\mathrm{c}}$ & $4,65^{\mathrm{c}}$ & $60,01^{\mathrm{a}}$ & $653^{\mathrm{e}}$ \\
DDG & $\mathrm{U} 2$ & $87,15^{\mathrm{c}}$ & $1,37^{\mathrm{f}}$ & $41,71^{\mathrm{b}}$ & $3,76^{\mathrm{c}}$ & $60,43^{\mathrm{a}}$ & $672^{\mathrm{e}}$ \\
DDG & $\mathrm{U} 4$ & $89,55^{\mathrm{b}}$ & $1,49^{\text {ef }}$ & $43,92^{\mathrm{b}}$ & $7,90^{\mathrm{b}}$ & $57,68^{\mathrm{ab}}$ & $486^{\mathrm{f}}$ \\
DDGS & $\mathrm{U} 1$ & $93,09^{\mathrm{a}}$ & $4,68^{\mathrm{c}}$ & $16,15^{\mathrm{e}}$ & $6,64^{\mathrm{b}}$ & $49,58^{\mathrm{ab}}$ & $1107^{\mathrm{b}}$ \\
HP-DDGS & $\mathrm{U} 1$ & $93,50^{\mathrm{a}}$ & $2,94^{\mathrm{d}}$ & $51,67^{\mathrm{a}}$ & $14,52^{\mathrm{a}}$ & $39,75^{\mathrm{bc}}$ & $485^{\mathrm{f}}$ \\
Solúvel & $\mathrm{U} 2$ & $5,19^{\mathrm{g}}$ & $5,70^{\mathrm{b}}$ & $26,53^{\mathrm{d}}$ & $6,46^{\mathrm{bc}}$ & $3,88^{\mathrm{d}}$ & - \\
Probabilidade & & $<0,0001$ & $<0,0001$ & $<0,0001$ & $<0,0001$ & $<0,0001$ & $<0,0001$ \\
CV $(\%)$ & & 0,73 & 2,17 & 5,28 & 9,53 & 9,53 & 5,07 \\
\hline
\end{tabular}

*Nível de significância <0,01 (Médias seguidas por letras diferentes na coluna apresentam diferenças pelo teste de Tukey); Teores de MM, PB, EE e FDN são expressos com base em matéria seca. CV = Coeficiente de variação. Fonte: Autores (2021).

Tabela 3 - Valores de energia bruta (EB), energia digestível (ED), energia metabolizável (EM) e energia líquida (EL) de diferentes coprodutos $(\mathrm{kcal} / \mathrm{kg})$.

\begin{tabular}{cccccc}
\hline Fontes & Origem & EB & ED & EM & EL \\
\hline WDG & $\mathrm{U} 1$ & $4786^{\mathrm{cd}}$ & $3619^{\mathrm{a}}$ & $3390^{\mathrm{a}}$ & $2218^{\mathrm{b}}$ \\
WDG & $\mathrm{U} 2$ & $4674^{\mathrm{de}}$ & $3040^{\mathrm{b}}$ & $2837^{\mathrm{c}}$ & $1761^{\mathrm{d}}$ \\
WDG & $\mathrm{U} 3$ & $4796^{\mathrm{cd}}$ & $3165^{\mathrm{b}}$ & $2842^{\mathrm{bc}}$ & $1959^{\mathrm{c}}$ \\
DDG & $\mathrm{U} 3$ & $4753^{\mathrm{cde}}$ & $2974^{\mathrm{b}}$ & $2705^{\mathrm{c}}$ & $1786^{\mathrm{d}}$ \\
DDG & $\mathrm{U} 2$ & $4706^{\mathrm{e}}$ & $2944^{\mathrm{b}}$ & $2632^{\mathrm{c}}$ & $1726^{\mathrm{d}}$ \\
DDG & $\mathrm{U} 4$ & $4934^{\mathrm{b}}$ & $3114^{\mathrm{b}}$ & $2765^{\mathrm{c}}$ & $2020^{\mathrm{b}}$ \\
DDGS & $\mathrm{U} 1$ & $4808^{\mathrm{c}}$ & $3169^{\mathrm{b}}$ & $3028^{\mathrm{abc}}$ & $1986^{\mathrm{b}}$ \\
HP-DDGS & $\mathrm{U} 1$ & $5269^{\mathrm{a}}$ & $3625^{\mathrm{a}}$ & $3163^{\mathrm{ab}}$ & $2616^{\mathrm{a}}$ \\
Probabilidade & & $<0,0001$ & $<0,0001$ & $<0,0001$ & $<0,0001$ \\
CV $(\%)$ & & 0,53 & 4,90 & 5,00 & 4,88 \\
\hline
\end{tabular}

*Nível de significância <0,01 (Médias seguidas por letras diferentes na coluna apresentam diferenças pelo teste de Tukey); Valores com base em matéria seca. CV = Coeficiente de variação. Fonte: Autores (2021). 
Figura 1 - Lisina total e lisina digestível de diferentes coprodutos de etanol.

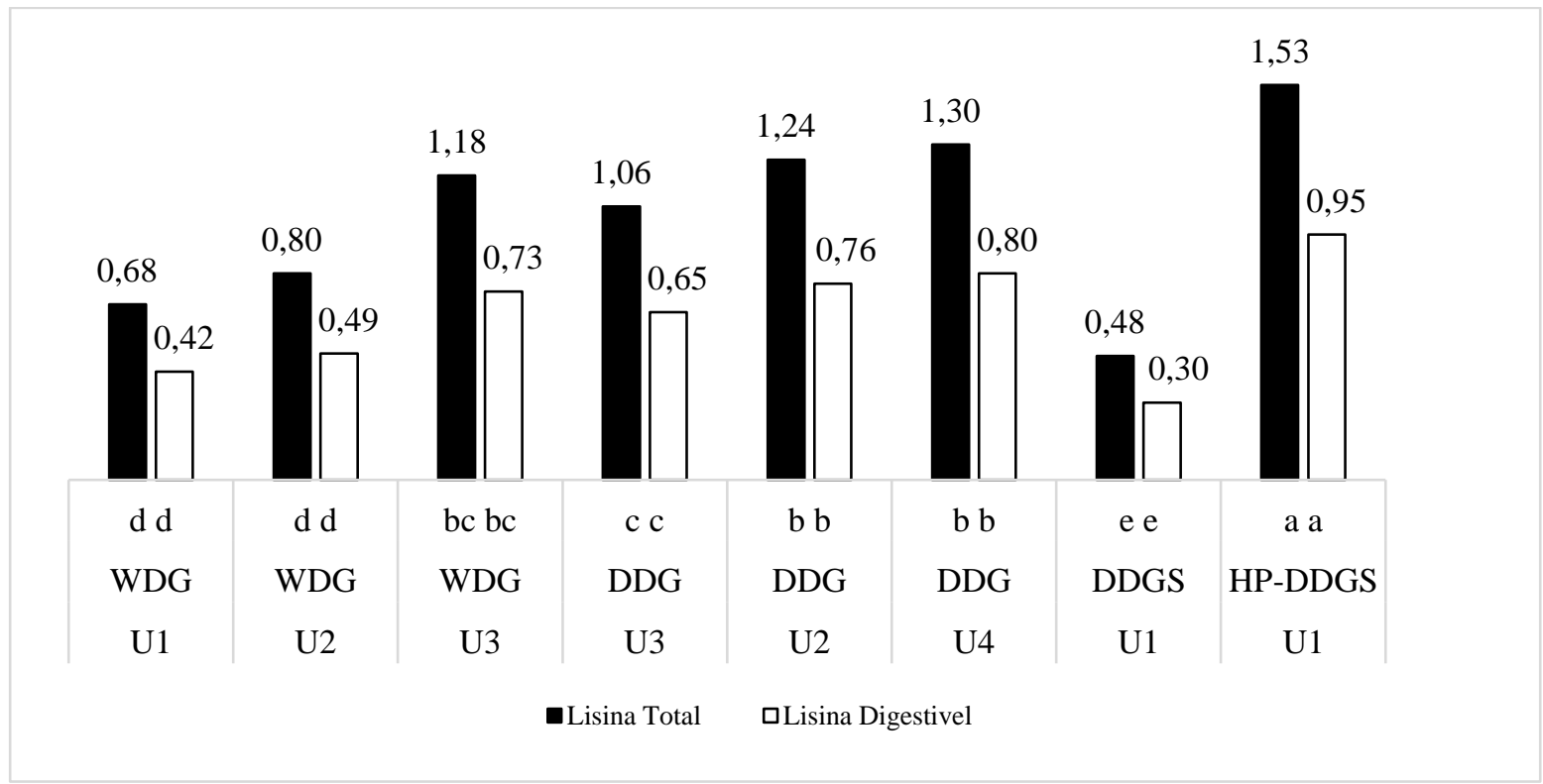

Valores com base na matéria seca. Colunas contendo letras diferentes apresentam diferenças pelo teste de Tukey a $<0,01$ de significância. Fonte: Autores (2021).

Estes resultados estão relacionados principalmente aos processos de fabricação utilizados em cada usina de etanol. Processos de moagem, fermentação, secagem, separação de fibras, inclusão de solúveis e retirada de óleo são exemplos de etapas realizadas distintamente nas usinas no intuito de otimizar o uso e caracterização dos coprodutos de acordo com a especificidade e interesse. A partir disso, diferentes equipamentos, fluxos de produção e uso de aditivos também podem influenciar na composição final dos coprodutos. Variações na composição dos grãos utilizados como matéria-prima podem estar ligadas à diversidade dos resultados obtidos.

Variabilidade na composição química também foi demonstrada em estudos prévios que registraram valores médios de 89, 4, 31, 11, 40\% (Anderson et al., 2012), 87, 5, 31, 11, 37\% (Kerr et al., 2013), 89, 5, 32, 9, 37\% (Li et al., 2015) e 90, 4, 30, 10, 47\% (Wu et al., 2016a) para MS, MM, PB, EE e FDN ao analisarem 6, 11, 25 e 3 coprodutos de etanol, respectivamente. Ao realizar meta-análise com 90 DDGS de milho, Zeng et al. (2017) registraram coeficientes de variação de 24,9; 8,6; 36,3; 13,4 e $17,8 \%$ para MM, PB, EE, FDN e lisina, respectivamente.

Apesar de todos os produtos analisados configurarem coprodutos da produção de etanol de milho, os resultados evidenciam a necessidade da caracterização e quantificação de componentes químicos, físicos e nutricionais de cada produto para formação da correta matriz nutricional e escolha de compra, formulação e confecção de ração para os suínos.

Os maiores teores de MS foram observados nos DDG's seguidos pelos WDG's e solúveis $(\mathrm{P}<0,01)$. A passagem do coproduto pela etapa final de secagem aumenta a concentração de MS nos DDG's, tornando-o mais adequado para armazenamento, transporte e uso em fabricação de rações. Em sentido oposto, os solúveis raramente são comercializados justamente por apresentarem baixo teor de MS, sendo indicada sua inclusão em outros coprodutos durante o processo fabril. O DDGS e HP-DDGS provenientes da U1 e o DDG da U3 foram os que apresentaram maiores valores de MS (P<0,01).

O WDG da U1 seguido pelos solúveis e pelo DDGS da U1 foram os coprodutos com maiores teores de $\mathrm{MM}(\mathrm{P}<0,01)$, podendo ser indicativos de maiores concentrações de nutrientes como cálcio e fósforo. Este aporte de minerais também é uma virtude dos coprodutos, especialmente quanto ao fósforo que é um nutriente bastante oneroso e de limitada disponibilidade entre os produtos de origem vegetal. 
Na determinação do teor de PB observou-se que o HP-DDGS apresentou o maior valor ( $\mathrm{P}<0,01)$ enquanto os DDG's da U4 e U2 e o WDG da U3 não apresentaram diferença entre si ( $P>0,01)$, sendo que o DDGS da U1 apresentou o menor teor entre os coprodutos analisados $(\mathrm{P}<0,01)$. A concentração de $\mathrm{PB}$ nos coprodutos de etanol é um atributo importante tendo em vista a intenção de utilização destes em substituição ao farelo de soja em se tratando de ingrediente fornecedor de aminoácidos nas dietas animais. A utilização do amido presente nos grãos para produção do etanol acrescido pelas leveduras no processo fermentativo, resulta em aumento da concentração da fração proteica nos coprodutos, agregando valor nutricional e comercial. Salientando que as leveduras possuem alto teor de proteína (37,2\%, Rostagno et al., 2017) e consequentemente os coprodutos também passam a ter maiores teores de PB.

A PB do HP-DDGS apresentou teor maior que o relatado por Adeola e Ragland (2016), enquanto a média encontrada entre as fontes de WDG foi próxima ao valor descrito por Lardy e Anderson (2014) e maior do que a relatada por Rosentrater et al. (2019). Quando comparado o valor médio de PB das fontes de DDG é superior ao relatado por Adeola e Ragland, (2016). Enquanto que o DDGS apresentado neste trabalho possui valor inferior ao encontrado por Anderson et al. (2012), Kerr et al. (2013), Li et al. (2015) e Wu et al. (2016a).

As maiores fontes de variação do teor de PB são os conteúdos das matérias-primas, embora a digestibilidade dos aminoácidos, em especial a lisina, e a ligação de compostos nitrogenados à fibra, sejam bastante influenciados pelo calor no processo de secagem (Böttger \& Südekum, 2018). Valores de composição química e nutricional obtidos neste estudo são distintos de outros uma vez que o milho cultivado no Brasil é mais vítreo quando comparado ao produzido nos EUA (Santos et al., 2015). Fatores como cozimento, sacarificação ou desidratação da vinhaça fina, inclusão dos solúveis, adição de uréia ou amônia, moagem dos grãos e biomassa de leveduras também influenciam o conteúdo nitrogenado dos coprodutos de etanol de grãos (Böttger \& Südekum, 2018).

O quantitativo de EE encontrado nos ingredientes representa a concentração de lipídeos e é referenciado como fonte e preditor dos valores de energia para suínos. O maior teor de EE foi registrado para HP-DDGS da U1 seguido pelos WDG’s da U1 e U3 ( $\mathrm{P}<0,01)$, DDG’s da U4 e U1 e solúveis que não se diferenciaram entre si ( $\mathrm{P}>0,01)$. Os menores valores de EE foram observados para WDG e DDG da U2 e DDG da U3 ( $\mathrm{P}<0,01)$. Os resultados obtidos caracterizam ampla diversidade nos teores de EE dos coprodutos brasileiros. Segundo NRC (2012), DDGS podem ser classificados com alto ( $>10 \%)$, médio ( $>6$ e $<9 \%$ ) ou baixo $(<4 \%)$ teor de EE.

Via de regra os ingredientes que possuem maior teor de proteína tendem a possuir menor EE, como observado no trabalho de Wu et al. (2016a) porém não foi o resultado encontrado para o HP-DDGS, que possui alta proteína e alto extrato etéreo, esse fato pode ter ocorrido devido a forma de produção e ao tipo de matéria prima utilizado (SMITH et al., 2015). O valor médio de EE para o WDG foi menor do que o referenciado por Lardy e Anderson (2014), enquanto a média para o DDG foi próxima a média de WDG, com menor teor que o encontrado por Adeola e Ragland (2016). O solúvel apresentou 6,46\% de EE, valor inferior à média (17\%) do solúvel condensado apresentado por Lardy e Anderson (2014).

Os WDG's e DDG's não apresentaram diferença entre si nos teores de FDN (P>0,01), à exceção do WDG da U1. O HP-DDGS apresentou teor intermediário enquanto os solúveis apresentaram o menor valor $(\mathrm{P}<0,01)$. A média de FDN entre as fontes de DDG foi superior ao encontrado por Adeola e Ragland (2016), assim como o DDGS apresentou valor superior ao observado por Anderson et al. (2012), Kerr et al. (2013), Li et al. (2015) e Wu et al. (2016a).

Teores de FDN refletem principalmente a concentração de fibras insolúveis dos alimentos e estão relacionados à diminuição da digestibilidade da PB (Saqui-Salces et al., 2017), MS, EB e aminoácidos (Acosta et al., 2020) em dietas para suínos. A fração de fibra insolúvel pode aprisionar nutrientes associados à matriz da parede celular e limitar a exposição à atuação de enzimas digestivas (Acosta et al., 2020). Embora a presença de altos níveis de fibra possa comprometer a digestibilidade da dieta e diluir os níveis de energia, a mesma é importante pois garante saúde intestinal, motilidade e bem- 
estar animal para os suínos (Gomes et al., 2005). Processos de separação de fibras antes da fermentação são empregados por algumas usinas na intenção de aumentar o rendimento de produção de etanol, gerando coprodutos distintos, um com elevado teor de fibras e outro baixo em fibras e alta concentração de proteína, como pode se observar nos resultados deste estudo.

O maior teor de TP foi observado no WDG da U1, seguido pelo DDGS da mesma usina, WDG da U3, WDG da U2 e DDG's destas últimas usinas $(\mathrm{P}<0,01)$. O menor valor de TP foi registrado para HP-DDGS $(\mathrm{P}<0,01)$. O TP apresentou grande amplitude entre as amostras, corroborando com os resultados de Liu (2008) ao analisar diversas fontes de DDGS. O TP dos coprodutos é consequência direta do processo de moagem empregado no início do processo de fabricação do etanol e esta condicionado ao procedimento de cada usina. A caracterização física de TP está relacionada ao aproveitamento dos alimentos uma vez que apresenta relação inversa com área específica e ação de enzimas digestivas, o que influencia na digestibilidade da dieta com observado por Saqui-Salces et al. (2017) ao registrarem maior, digestibilidade da MS, FDN e PB, eficiência alimentar e ganho de peso diário em suínos alimentados com rações com fino $(374 \mu \mathrm{m}) \mathrm{TP}$ em comparação àqueles com tamanho grosseiro $(631 \mu \mathrm{m})$ em dietas contendo coprodutos para suínos.

O HP-DDGS apresentou maior teor de EB, seguido pelo DDG da U4 (P<0,01) enquanto WDG's da U1 e U3 e DDG's da U3 e DDGS da U1 não se distinguiram entre si $(\mathrm{P}>0,01)$ e menores valores para WDG e DDG da U2 e DDG da U3 $(\mathrm{P}<0,01)$. O HP-DDGS e WDG da U1 apresentaram maiores valores de ED em relação aos demais $(\mathrm{P}<0,01)$ que não diferiram entre si $(\mathrm{P}>0,01)$. De maneira similar, HP-DDGS, DDGS e WDG da U1 apresentaram maiores valores de EM em relação ao WDG e DDG da U2 e DDG's da U4 e U3, que não diferiram entre si. Quanto aos valores de EL, o HP-DDGS foi maior que os demais, seguido pelo WDG e DDGS da U1, DDG da U4, seguidos pelo WDG e DDG da U2 e DDG da U3 (P<0,01). Coprodutos como HP-DDGS expressam altos valores de energia em função de altos teores de PB e EE e baixos teores de FDN.

Com exceção do WDG e HP-DDGS da U1, todos os coprodutos apresentaram teores de EB, ED e EM abaixo dos valores médios de estudos prévios evidenciaram valores médios de 5420, 4029, $3790 \mathrm{kcal} / \mathrm{kg}$ (Anderson et al., 2012), 5024, 3634, $3240 \mathrm{kcal} / \mathrm{kg}$ (Kerr et al., 2013), 5109, 3661, $3475 \mathrm{kcal} / \mathrm{kg}$ (Li et al., 2015), mas próximos de 4750 (EB) e 3283 kcal/kg (EM) (Wu et al., 2016a), respectivamente.

O teor de EM do DDGS de origem brasileira avaliado por Corassa et al. (2017) foi de 3.668 e $3.213 \mathrm{kcal} / \mathrm{kg}$ pelo método de coleta total e pela técnica do marcador, respectivamente. Por outro lado, Stuani et al. (2016), em uma abordagem analítica, compilaram resultados de diversos estudos e obtiveram um valor médio de $3.695 \mathrm{kcal} / \mathrm{kg}$ para EM. Outro estudo investigando DDGS de milho produzido no Brasil, Corassa et al. (2019) registraram valores de 3477 e $3761 \mathrm{kcal} / \mathrm{kg}$ para ED e 3277 e $3609 \mathrm{kcal} / \mathrm{kg}$ para EM com 20 e $40 \%$ de inclusão do ingrediente na dieta, respectivamente.

Os teores de lisina total e digestível foram maiores no HP-DDGS, seguidos pelo WDG da U3 e DDG's da U2 e U4 e, sendo estes últimos maiores que o DDG da U3 seguidos pelos WDG's da U1 e U2 com o DDGS da U1 com os menores valores $(\mathrm{P}<0,01)$. Por ser considerado o principal aminoácido na nutrição de suínos, os teores de lisina encontrados nos ingredientes podem dar referência do aporte dos demais aminoácidos e da capacidade destes em atender às exigências dos animais. Todos os valores de lisina digestível encontrados no presente estudo foram inferiores aos valores médios de 1,17 (Anderson et al., 2012), 1,11 (Kerr et al., 2013); e 1,04\% (Wu et al., 2016a) enquanto o HP-DDGS foi o coproduto que se aproximou de $0,91 \%$ registrado por $\mathrm{Li}$ et al. (2015).

\section{Considerações Finais}

Há diversidade na composição dos coprodutos de etanol de milho devido à matéria prima e processos de fabricação distintos entre as usinas brasileiras. As caracterizações químicas, físicas e nutricionais sugerem potencial de uso dos coprodutos em dietas para suínos. Entre os coprodutos investigados, o HP-DDGS apresentou maior concentração de componentes nutricionais e com maior aptidão para uso em dietas para suínos. 


\section{Referências}

Acosta, J. A., Stein, H. H. \& Patience, J.F. (2020). Impact of increasing the levels of insoluble fiber and on the method of diet formulation measures of energy and nutrient digestibility in growing pigs. Journal of Animal Science, 98 (6), 1-9. https://doi:10.1093/jas/skaa130

Adeola, O. \& Ragland, D. (2016). Comparative ileal amino acid digestibility of distillers' grains for growing pigs. Animal Nutrition, 02(04), 262-266. https://doi:10.1016/j.aninu.2016.07.008

Anderson, P. V., Kerr, B. J., Weber, T. E., Ziemer, C. J. \& Shurson, G. C. (2012). Determination and prediction of digestible and metabolizable energy from chemical analysis of corn coproducts fed to finishing pigs. Journal of Animal Science, 90(04), 1242-1254.https://doi:10.2527/jas.2010-3605

AOAC. Association of Official Analytical Chemists. (2005). "Official Methods of Analysis.” (18th ed.), OAC.

ABPA. Associação Brasileira de Proteína Animal (2019). Relatório anual 2019.

Böttger, C. \& Südekum, K. H. (2018). Review: protein value of distillers dried grains with solubles (DDGS) in animal nutrition as affected by the ethanol production process. Animal Feed Science and Technology, 244, 11-17. https://doi:10.1016/j.anifeedsci.2018.07.018

Chatzifragkou, A., Kosik, O., Prabhakumari, P. C.; Lovegrove, A., Frazier, R.A., Shewry, P. R., \& Charalampopoulos, D. (2015). Biorefinery strategies for upgrading Distillers' Dried Grains with Solubles (DDGS). Process Biochemistry, 50(12), 2194-2207. https://doi:10.1016/j.procbio.2015.09.005

Corassa, A., Lautert, I. P. A. S., Pina, D. S., Kiefer, C., Ton, A. P. S., Komiyama, C. M., Amorim, A. B. \& Teixeira, A.O. (2017). Nutritional value of Brazilian distillers dried grains with solubles for pigs as determined by different methods. Revista Brasileira de Zootecnia, 46(9), 740-746. https://doi:10.1590/s1806-92902017000900005

Corassa, A., Lautert, I.P.A.S, Ton, A.P.S., Kiefer, C., Brito, C.O., Sbardella, M. \& Souza, H.C. (2021). Viability of Brazilian distillers dried grains with solubles for pigs. Semina: Ciências Agrárias, 42(3), 1159-1174. http://dx.doi.org/10.5433/1679-0359.2021v42n3p1159

Corassa, A., Stuani, J.L., Ton, A. P. S. , Kiefer, C. , Sbardella, M. , Brito, C. O. , Amorim, A. B. \& Goncalves, D. B. C. (2019). Nutritional value of distillers dried grains with solubles from corn and sorghum and xylanase in diets for pigs. Revista Brasileira de Zootecnia, 48 , e20190012. https://doi:10.1590/rbz4820190012

CONAB. Companhia Nacional de Abastecimento. (2020). Acompanhamento da safra brasileira - Cana-de-açúcar. Conab,. 58p.

Fantini, C. C., Brumatti, R. C., Lopes, B. F. C. L., Gaspar, A. O., Kiefer, C. \& Corassa, A. (2021). Economic evaluation of the use of distillers dried grains with solubles in swine feeding. Research, Society and Development, 10 (7), e0110716082. http://dx.doi.org/10.33448/rsd-v10i7.16082

Gomes, J. D. F., Fukushima, R. S., Putrino, S. M., Grossklaus, C. \& Lima, G. J. M. M. (2005). Efeitos do incremento da fibra em detergente neutro na dieta de suínos sobre a morfologia dos órgãos digestivos e não digestivos. Brazilian Journal of Veterinary Research and Animal Science. 43(02), 202-209. file:///C:/Users/Corassa/Downloads/26500-Article\%20Text-30771-1-10-20120619\%20(1).pdf

Kerr, B. J., Dozier, W. A. \& Shurson, G. C. (2013). Effects of reduced-oil corn distillers dried grains with solubles composition on digestible and metabolizable energy value and prediction in growing pigs. Journal of Animal Science. 91(7), 3231-3243. http://doi: 10.2527/jas.2013-6252.

Lardy, G. \& Anderson, V. (2014). Feeding Coproducts of the Ethanol Industry to Beef Cattle. NDSU Extension Service, August 2014.

Leite, R. C. C. \& Leal, M. R. L. V. (2007). O biocombustível no Brasil. Revista Novos Estudos CEBRAP. 78, 15-21. https://doi.org/10.1590/S010133002007000200003

Li, P., Li, D. F., Zhang, H. Y., Li, Z. C., Zhao, P. F., Zeng, Z. K., Xu, X. \& Piao, X. S. (2015). Determination and prediction of energy values in corn distillers dried grains with solubles sources with varying oil content for growing pigs. Journal of Animal Science. 93(7), 3458-3470. https://doi.org/10.2527/jas.20148782

Liu, K. (2008). Particle size distribution of distillers dried grains with solubles (DDGS) and relationships to compositional and color properties. Bioresource Technol. 99(17), 8421-8428. https://DOI: 10.1016/j.biortech.2008.02.060

NRC. National Research Concil. (2012). Nutrient Requirements of Swine (11a ed.). Publishing company: National Academy Press, Washington, D. C.

RFA. Renewable Fuels Association. Focus forward: 2020 ethanol industry outlook. RFA, 2020.

Rosentrater, K., Kalscheur, K., Garcia, A. \& Wright, C. (2019). Fuel ethanol coproducts for livestock diets. Distillers Grains Technology Council. Recuperado de < https://distillersgrains.org/distillers-grains/ >

Rostagno, H. S., Albino, L. F. T., Hannas, M. I., Donzele, J. L., Sakomura, N. K., Perazzo, F. G., Saraiva, A., Teixeira, M. L., Rodrigues, P. B., Oliveira, R. F., Barreto, S. L. T. \& Brito, C. O. (2017). Tabelas brasileiras para aves e suínos. Composição de alimentos e exigências nutricionais (4a ed.). Departamento de Zootecnia. UFV.

Santos, F. A. P., Batistel, F. \& Souza, J. (2015). Processamento aumenta aproveitamento do milho e eficiência de rações animais. Visão Agrícola 13, 153-153. https://www.esalq.usp.br/visaoagricola/sites/default/files/VA_13_Industrializacao-box2.pdf

Saqui-Salces, M., Luo, Z., Urriola, P. E., Kerr, B. J. \& Shurson, G. C. (2017). Effect of dietary fiber and diet particle size on nutrient digestibility and gastrointestinal secretory function in growing pigs. Journal of Animal Science.95:2640-2648. https://doi:10.2527/jas2016.1249

Silva, A. L. \& Castañeda-Ayarza, J. A. (2021). Macro-environment analysis of the corn ethanol fuel development in Brazil. Renewable and Sustainable Energy Reviews, 135, 110387. https://doi.org/10.1016/j.rser.2020.110387 
Research, Society and Development, v. 10, n. 13, e105101321031, 2021

(CC BY 4.0) | ISSN 2525-3409 | DOI: http://dx.doi.org/10.33448/rsd-v10i13.21031

Smith, B., Hassen, A., Hinds, M., Rice, D., Jones, D., Sauber, T., Iiams, C., Sevenich, D., Allen, R., Owens, F., Mcnaughton, J. \& Parsons, C. (2015). Predicting the digestible energy of corn determined with growing swine from nutrient composition and cross-species measurements. Journal of Animal Science. 93(3), 1025-1038. https://doi: 10.2527/jas.2014-7807

Stein, H. H. (2008). Use of distllers co-products in diets fed to swine, 79-97. In: Babcock, B. A., Hayes, D. J., Lawrence, J. D. Using distillers grains in the U.S. and international livestock and poultry industries. 79-97. 〈https://www.card.iastate.edu/products/books/distillers_grains/pdfs/chapter4.pdf>

Stuani, J. L., Corassa, A. \& Silva, I. P. A. (2016). Caracterização nutricional e uso de DDGS em dietas para suínos em crescimento e terminação - abordagem analítica. Nativa, 4, 116-120. https://doi.org/10.31413/nativa.v4i2.2988

Urriola, P. E., LI, M., Kerr, B. J. \& Shurson, G. C. (2014). Evaluation of prediction equations to estimate gross, digestible, and metabolizable energy content of maize dried distillers grains with solubles (DDGS) for swine based on variable chemical composition. Animal Feed Science and Technology, 198, 196-202. https://doi:10.1016/j.anifeedsci.2014.09.006

Wu, F., Johnston, L. J., Urriola, P. E., Hilbrands, A. M. \& Shurson, G. C. (2016a). Evaluation of ME predictions and the impact of feeding maize distillers dried grains with solubles with variable oil contenton growth performance, carcass composition, and pork fat quality of growing-finishing pigs. Animal Feed Science and Technology. 213, 128-141.https://doi.org/10.1016/j.anifeedsci.2016.01.013

Wu, F., Johnston, L. J., Urriola, P. E., Hilbrands, A. M. \& Shurson, G. C. (2016b). Effects of feeding diets containing distillers' dried grains with solubles and wheat middlings with equal predicted dietary net energy on growth performance and carcass composition of growing-finishing pigs. Journal of Animal Science. 94(1), 144-154. https://doi.org/10.2527/jas.2015-9592

Zanotto, D. L. \& Bellaver, C. (1996). Método de determinação da granulometria para uso em rações de suínos e aves CT/215/EMBRAPA-CNPSA. Recuperado de < http://www.cnpsa.embrapa.br/sgc/sgc_publicacoes/cot215.pdf>

Zeng, Z. K., Shurson, G. C. \& Urriola, P. E. (2017). Prediction of the concentration of standardized ileal digestible amino acids and safety margins among sources of distillers dried grains with solubles for growing pigs: A meta-analysis approach. Animal Feed Science and Technology, 231, 150-159. https://doi.org/10.1016/j.anifeedsci.2017.07.010 